\title{
Structures of Turbulent Vortices in Floodplains of Compound Channels
}

\begin{abstract}
Alfonsas Rimkus
Water Research Institute of Aleksandras Stulginskis University, LT-58102, Vilainiai, Kedainiai, Lithuania, e-mail: alfonsas.rimkus@hidro.lzuu.lt
\end{abstract}

(Received May 11, 2016; revised February 08, 2017)

\begin{abstract}
In turbulent flows, several kinds of vortices and their structures are developed. After physical summation al velocities created by them, they intertwine, and the picture obtained from their measurement chronograms is mostly chaotic. Consequently, these structures become masked. It is therefore very difficult to investigate them and despite many studies performed so far, they have not been sufficiently explored. Favorable conditions for investigations of velocity measurement chronograms occurred when sufficient long intervals were observed, in which the above mentioned masking is minimal. In such intervals, the investigation of turbulent vortices and their structures becomes possible, and it has been performed to some degree. The results of these investigations of the compound channel were already discussed in two previously published articles. The first describes all vortices and their structures in the whole water flow of the compound channel. The second contains a detailed analysis of vortices and their structures in the central vertical of the main channel. This article, discuss investigations of coherent structures formed in the floodplain.
\end{abstract}

Key words: hydraulic investigations, coherent structures, measurement analysis

\section{Introduction}

The velocities of turbulent vortices and their structures, developed in the flow, get tangled by their summing in the flow. Thus these structures become masked and invisible, which makes it difficult to investigate them. That is why despite many studies on turbulent flows (Vanoni and Nomicos 1959, Klaven 1966, Grishanin 1969, Nezu and Azuma 2004), their structure has not yet been sufficiently explored. Another difficulty for these investigations is the fact that, when a detailed picture of measured velocities is necessary the usually employed PIV method, provides a photograph that can record only a short interval of the flow, which cannot include even a single macro whirlpool. Thus, these whirlpools cannot be investigated. Therefore, in this study, turbulence was investigated by analyzing instantaneous velocity measurements carried out by 
3D means. Such measurements take several minutes at each point, during which all the vortices recur many times and can be studied.

It has been revealed by many previous investigations that there are two main types of turbulent vortices, i.e. vortices, generated near the bottom, where critical velocity gradients are formed, and the macro whirlpools developed along the whole depth of flow. Their structure, investigated by Vanoni and Nomicos, is shown in Fig. 1.

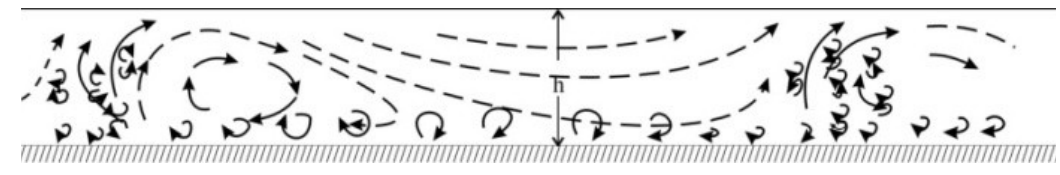

Fig. 1. Distribution of turbulent vortices according to Vanoni and Nomicos (1959)

The investigations analyzed in this work were carried out in the hydraulic laboratory of Warsaw Agricultural University in a $16 \mathrm{~m}$ long and $2.1 \mathrm{~m}$ wide concrete channel with a three-component acoustic Doppler velocity meter at a frequency of $25 \mathrm{~Hz}$. Each measurement lasted $6.0 \mathrm{~min}$ (9000 measurement steps). The slope of the channel bed was 0.05 . The water depth $H=28.3 \mathrm{~cm}$. The surface of the main channel bed was smooth (Manning roughness coefficient $n=0.011 \mathrm{~m}^{-1 / 3} \mathrm{~s}$ ); the floodplains and sloping banks were covered in cement with grains from 0.5 to $1 \mathrm{~cm}$ in diameter $\left(n=0.018 \mathrm{~m}^{-1 / 3} \mathrm{~s}\right)$. The cross-section of the channel and distribution of measurement verticals are shown in Fig. 2. The measurement points in the verticals were distributed at distances of 1 or $2 \mathrm{~cm}$. Investigations of these data were first performed by their authors (Czernuszenko et al 2007, Czernuszenko and Rowinski 2008). They studied turbulent intensities, Reynolds stresses, autocorrelation function, turbulent scales and energy spectra.

The results of the author's earlier investigations of these data have been published in two articles (Rimkus 2012, 2015). The first describes all the vortex structures in the entire model. The second presents a detailed analysis of the detected coherent structures and of their interaction, studied along the axis of the main channel.

Earlier investigations have shown that single vortices, generated near the bottom, get displaced in the rows oblique to the flow direction. When the first vortex in a row is formed, it decreases longitudinal velocity near the bottom in a place favorable for vortex generation. When the first vortex has been moved by the flow, another vortex can be formed in its place. Thus, groups are formed.

The distances between vortices in their groups are not large. Therefore, only the oldest upper vortex can freely absorb a water volume by fluid viscosity and send it down to a neighboring vortex, which transports this volume to the next younger vortex. Thus, flow is created around the group. Consequently, these groups create the main kind of coherent structures in the flow. The best conditions for the formation of vortices and their groups exist in the middle between neighboring macro whirlpools; 


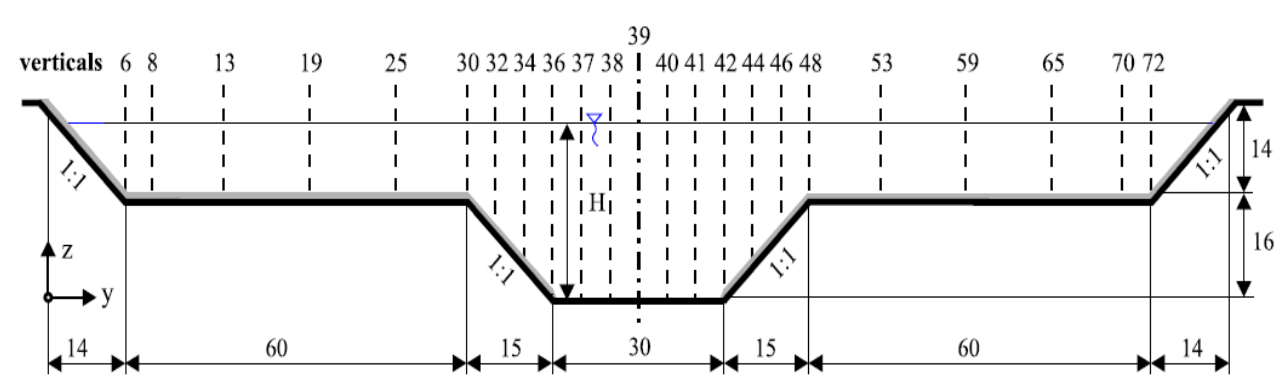

Fig. 2. Cross-section of the experimental channel and the distribution of measurement verticals. The measurements are given in $\mathrm{cm}$

here the largest flow velocity gradients are formed at the bottom. Therefore, the groups of vortices become thicker here and can influence one other. Thus, they form another kind of large coherent structures, namely accumulations of these groups. The process of their formation has already been discussed in detail in a previously published article (Rimkus 2015).

The previous investigations of these data have established that the physical summation of velocities formed by all vortices in the flow produces a chronogram that is not always suitable for necessary investigations. Therefore, all vortices and their groups, i.e. their velocity chronograms, must be separated, else they cannot be studied in detail. Separation methods have also already been discussed in the already published articles. Intensive interaction of macro whirlpools and bottom vortex structures has been stated.

In this article, vortex structures formed in the floodplain are discussed.

\section{The Structures of Vortices Formed in the Floodplain}

In floodplains, vortices and their structures are formed both near the bottom and near the floodplain banks. In both cases, they are also formed of two main kinds: (1) vortices generated and developed near the flow contours, i.e. at the bottom or near the banks and (2) large macro whirlpools developed across the whole depth of the flow or across the whole breadth of the floodplain. The velocities of all these vortices mostly get tangle by their summation, and then macro whirlpools become masked and invisible. The study of such velocity chronograms is very difficult. However, in the floodplains, as well as in the main channel, a short chronogram interval was found where this masking was almost absent, and the investigation of vortex structures was possible. Such an interval was found between the measurement points 5860 and 6200 in the $19^{\text {th }}$ vertical at a height of $4 \mathrm{~cm}$ from the bottom. This interval, favorable for investigation, is shown in Fig. 3, in which 3 macro whirlpools are visible (intervals 5870-6200, 6200-6540 and 6540-6810). In the other verticals, such intervals, that could be favorable for the study, were absent. Macro whirlpools are usually masked 
by long coherent structures formed by accumulations of vortex groups. This process is also analyzed in the aforementioned article (Rimkus 2015).

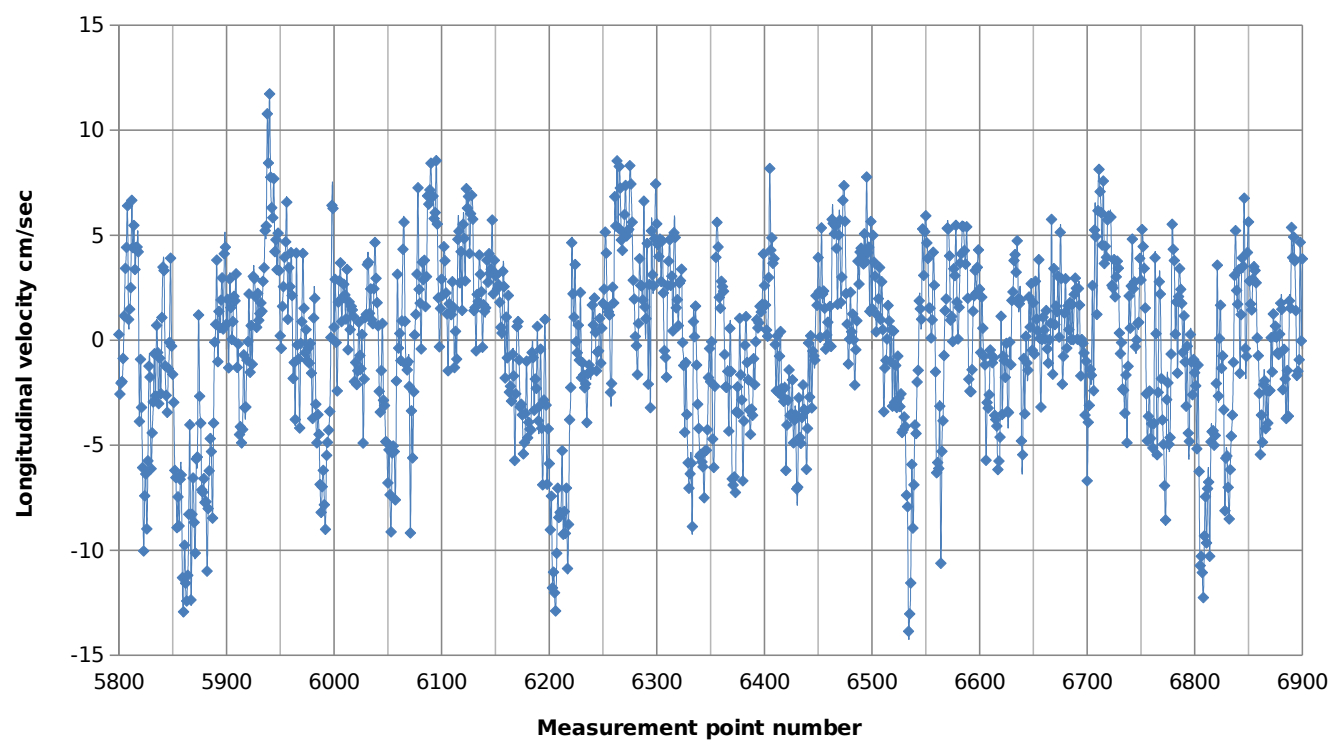

Fig. 3. Measurement chronogram of longitudinal velocities in vertical 19 at a height of $4 \mathrm{~cm}$ from the bottom

\subsection{Vortex Structures Formed at Floodplain Banks}

Similarly, as in the main channel, the vortices generated at the banks are also displaced in oblique rows in the width of floodplain. These groups develop a flow around them so they form the main coherent structures of vortices. In the intervals between the macro whirlpools, where the gradients of longitudinal velocities at the banks are greater, vortex groups are thicker and form the common field of vortices. Thus, the other kind of coherent structures is formed and in the floodplain. These large structures also develop velocities around them. Their velocity graph is included in the main measurement chronogram. All vortex structures with a vertical turning axis, formed at the banks, can be detected in the chronogram shown in Fig. 3, where 3 ordinary macro whirlpools are visible. Their length was calculated by computer procedures described in the aforementioned published article. It was received equal to 320 measurement steps. In Fig. 3, the limits of these macro whirlpools are visible at the local low velocity minimums. However, the distances between these minimums are not constant, only their average length is equal to 320 steps. That is because the groups of vortices are strong and their measurements and displacement are accidental, so they are able to change slightly the places of these minimums, or even to fill them, and thus to mask the 
limits of macro whirlpools. Each macro whirlpool contains 3 groups of bank vortices, the limits of which are also visible as velocity minimums.

It has been remarked that an interval, that was favorable for investigation and did not mask macro whirlpools, was found in a chronogram interval, in which vortex fluctuations are weaker. At intervals, where the fluctuations are stronger, the displacement of formed vortices is more accidental, and then these large vortex groups coincide with the limits of macro whirlpools and mask them. A decrease of water discharge reduces both the intensity and the dispersion of vortices and the masking lessens. Such changes in fluctuations along the flow are caused by voltage fluctuations in the electrical system, which change the water discharge flowing into the model and the intensity of vortices. It would be useful to perform velocity measurements with different water discharges and to investigate the influence of this phenomenon.

\subsection{The Vortex Structures Formed at the Floodplain Bottom}

The bottom vortices and their rows create a waving velocity graph. Its thick twists are visible along the velocity graph of vortex structures of bank velocities. The velocities of single vortices can be estimated from these twists by a method described in the aforementioned article. However the twists formed by bottom macro whirlpools are not visible. It occurred so, because the length of bottom macro whirlpools in the chronogram of longitudinal velocities is similar to the length of single bank vortices; so they mask one another and are not visible in Fig. 3. However, these macro whirlpools are well visible in the chronogram of the vertical velocity component, as bank vortices do not have a vertical velocity component. This chronogram is shown in Fig. 4. The length and amplitude of turning velocity change somewhat along the macro whirlpool of the floodplain. This is caused by the turning of horizontal macro whirlpools, which make the flow in the floodplain twist.

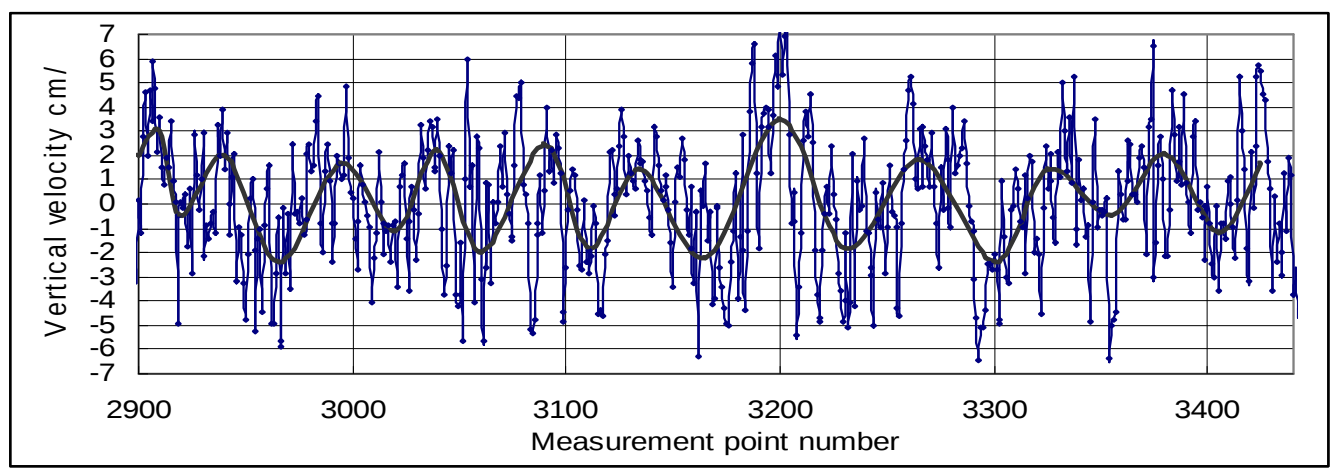

Fig. 4. The vertical component of bottom vortices and their groups (thin line) and of the bottom macro whirlpools (thick line) in the valley at a height of $4 \mathrm{~cm}$ in the $19^{\text {th }}$ vertical 
On the basis of the measurement data in Fig. 4, an approximate graph of longitudinal turning velocity was created for the bottom macro whirlpools. It is presented further in Fig. 6, where it is shown together with vortex structures that are left after separation of the abovementioned macro whirlpools from the graph of the measured velocities of all structures of turbulent vortices. This separation process is discussed further.

\subsection{Vortices Formed at the Contact of the Floodplain and the Main Channel Flows}

At the contact of the floodplain and the main channel flows, large gradients of longitudinal velocities are formed. They are a cause of intensive but relatively small vortices. Here, at this contact, there are no hard borders with favorable points at which series of vortices could be generated. Therefore, they do not form rows of groups. They are single but relatively intensive turbulent vortices. They grow quickly in the zone of high velocity gradients. Subsequently, they get into a zone further from the contact, where the gradients are lower, and they are suppressed. Thus, these vortices are active only in the area near the contact. They are visible only in the chronogram measured at vertical 30 .

\section{Separation of Velocity Chronograms of Various Structures of Vortices}

The need for the separation of all the various structures of vortices in the main channel was discussed in the articles mentioned before. The separation is necessary, because only then their properties can be investigated. Some errors can occur by calculation of the vortex structure characteristics, when the velocities of the measurements are directly employed, because they are somewhat distorted in the summation process. The separation helps to see how these structures develop and interact. The separation methods are described in the abovementioned articles.

In accordance with the methods, for the separation of each vortex structure, clearly visible twists of the chronogram were employed. The average velocity at all points of twist is equal to the sum of velocities formed by all the vortex structures left after the separation at the midpoint of the twist. The obtained points of the left structures are rare; so it was necessary to perform the interpolation between them in order to estimate the velocities of separated vortex structure at all measurement points. It was proved with parabolas of the $3^{\text {rd }}$ and the $5^{\text {th }}$ power. In the case of a higher power, the formed parabola sometimes did not go through all 6 points given to it. That was because of the peculiar form of the graph in some chronogram intervals. Thus, the $3^{\text {th }}$ power of the parabola was employed. For more exact interpolation, only the middle interval between the points of the parabola was used.

It is convenient to separate the horizontal macro whirlpools of the floodplain first. Then the velocity graph of all the other vortex structures twists around the straight zero axis instead of the macro whirlpool graph. For the separation, it was necessary 
to have a velocity graph of these whirlpools. The velocity graph of macro whirlpools in the main channel was formed in the velocity measurement chronogram, as they were clearly visible in the favorable interval of the graph. However, it was not so clear in the floodplain chronogram (Fig. 3), as the bank vortex groups in the floodplain are very large and therefore mask the macro whirlpools. The amplitude of the macro whirlpool was chosen in such way that the summary velocity graph of vortices, left after separation, would have the normal form. If the selected amplitude is too small, then the velocity minimum, formed between neighboring macro whirlpools, remains too low. If the amplitude is too large, the distinct velocity decrease is formed at the maximum of macro whirlpool velocities. Such abnormalities were absent in our case when the amplitude of macro whirlpool was $5.5 \mathrm{~cm} / \mathrm{sec}$. The chosen form of the macro whirlpool is shown in Fig. 5.

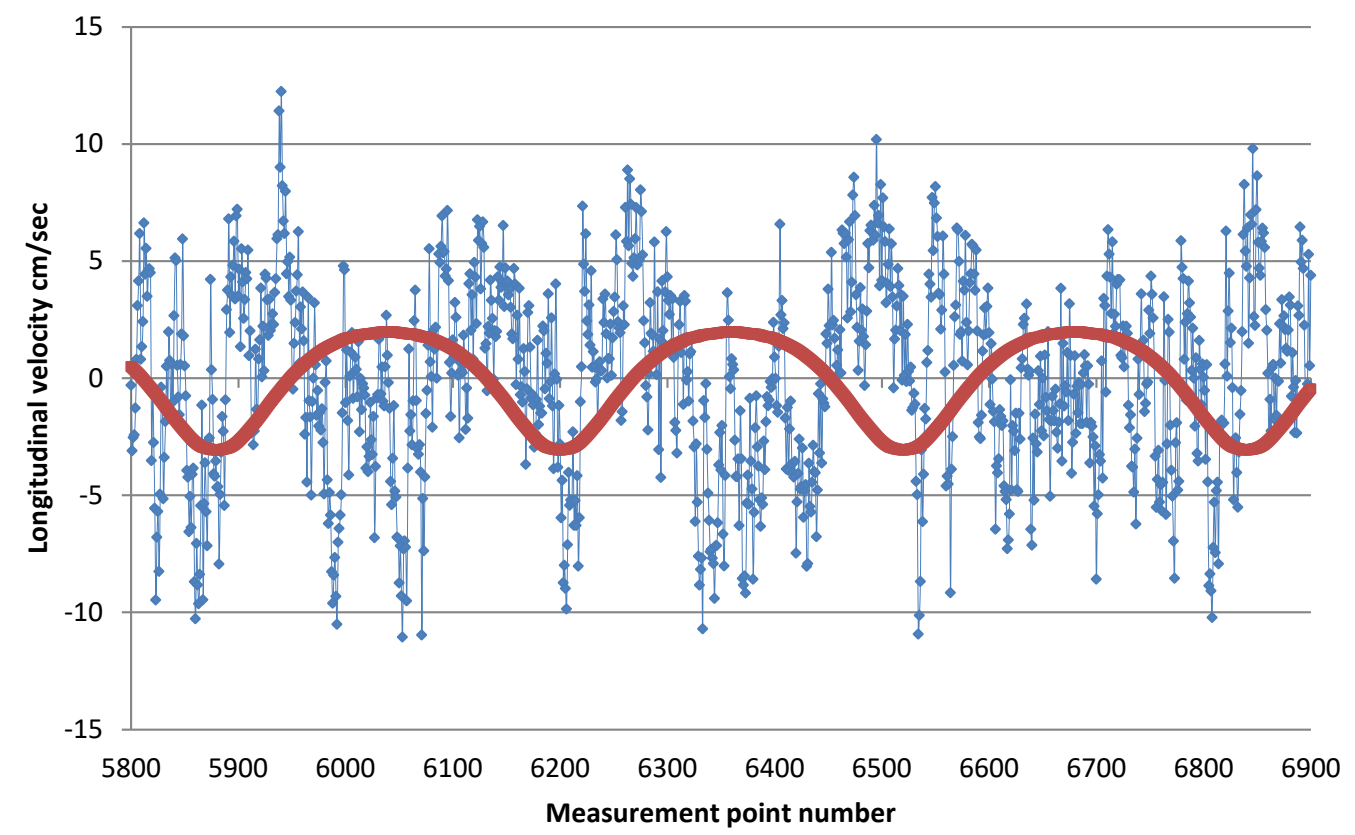

Fig. 5. Longitudinal velocities of horizontal macro whirlpools (thick line) and of the summed velocities of all the vortices left after the separation of horizontal macro whirlpools (thin line)

The bottom macro whirlpools are also convenient for further separation. However, as already mentioned, they are masked (Fig. 3) by the single bank vortices, which have similar length. The bottom macro whirlpools are not masked in the chronogram of vertical velocity components (Fig. 4). As it can be seen in this graph, the macro whirlpools are of somewhat exchanging length and amplitudes. That is because a turning horizontal macro whirlpool makes the flow twisting. Amplitudes of bottom macro whirlpools are the largest in the middle of the horizontal macro whirlpool as here the flow is parallel to the channel axis. On the basis of the visible measurement data, an 
average longitudinal velocity graph of the bottom macro whirlpools was created. The amplitudes of longitudinal velocities were also estimated in a way, that the graph, left after separation, would have the correct form. If the amplitudes were too large, the remaining graph became twisting. If they were too low, they made twists with the opposite sign. The obtained graph of longitudinal velocities is shown in Fig. 6.

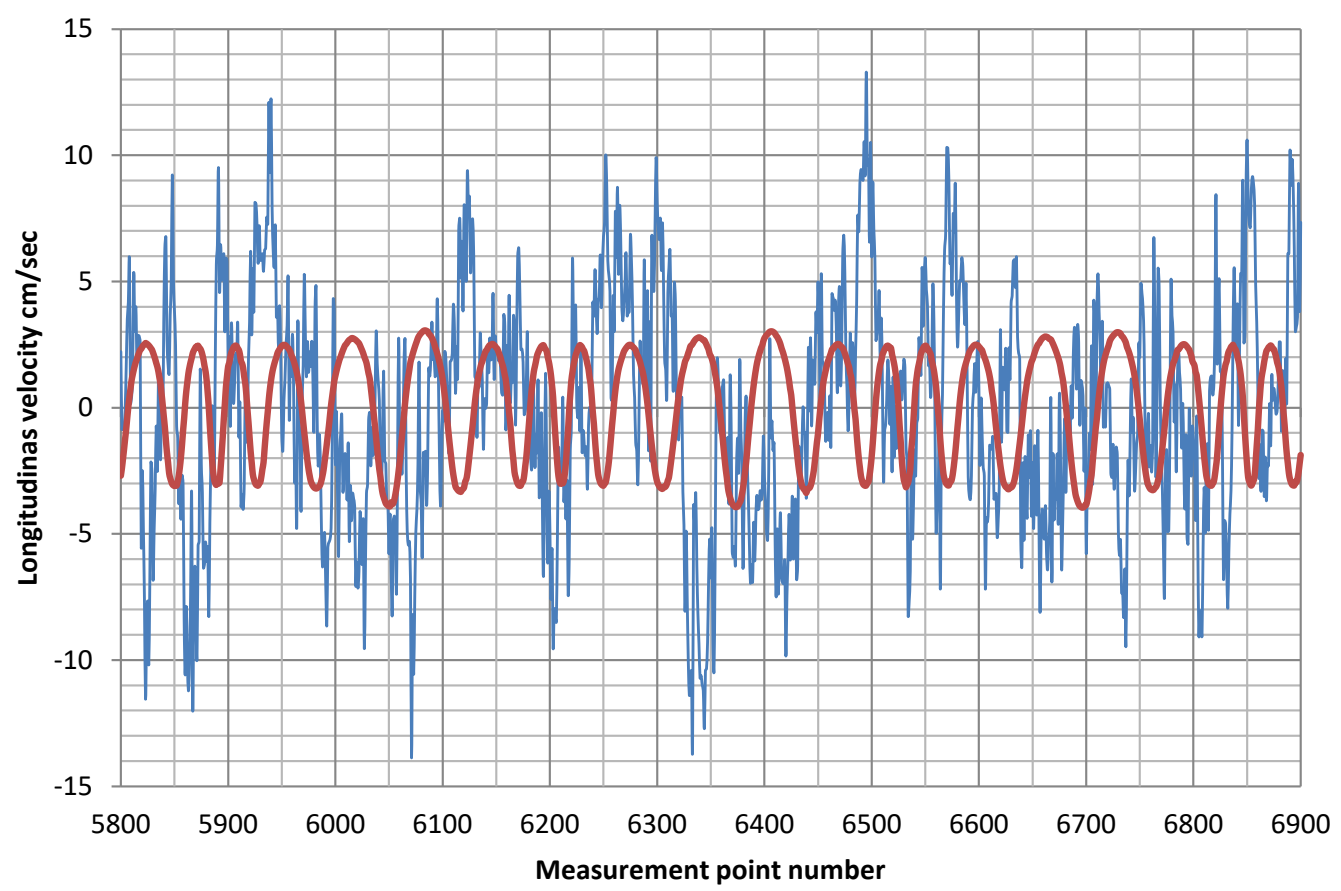

Fig. 6. Longitudinal velocities in the floodplain: bottom macro whirlpools (thick line) and summed velocities of all vortex structures left after the separation of bottom macro whirlpools and horizontal macro whirlpools in the floodplain (thin line)

Fig. 7 shows the further separated single bottom vortices, and Fig. 8 displays their separated groups. The obtained single vortices and their groups were very thick; therefore, they could be visible and shown only in a short interval (5900-6200) of measurements.

The velocity graph of accumulations, formed by the groups of bottom vortices, would also have been further separated. However, the frequency of these structures happened to be quite similar to the frequency of single bank vortices. Consequently, they could not be separated as different kinds. The separation gives the sum of velocities created by them both. Consequently, the obtained graph (Fig. 9) of summed longitudinal velocities cannot be used for their investigation and for calculation of the energy of these vortex types. For this purpose, these vortex types would have been separated; however, it occurred that this is impossible. Further separation of bank vor- 


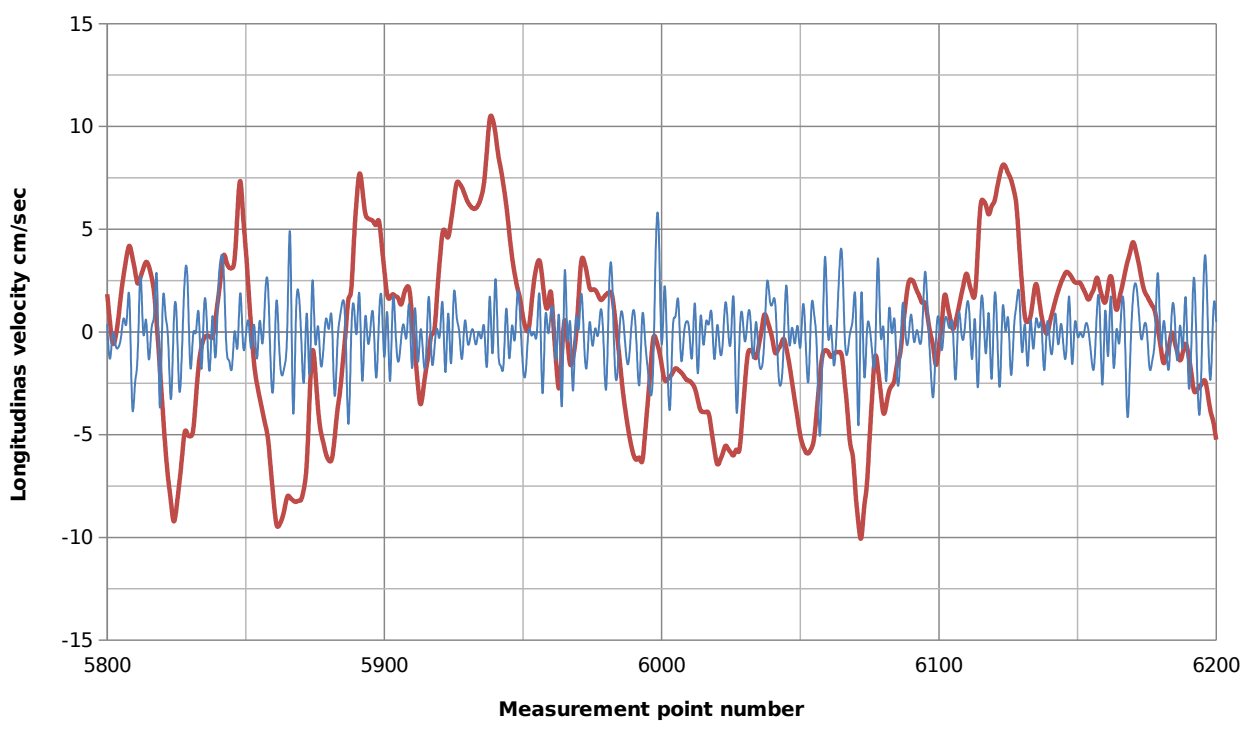

Fig. 7. Longitudinal velocity of separated single bottom vortices (thin line) and summed velocities that are left after the separation of vortex structures (thick line)

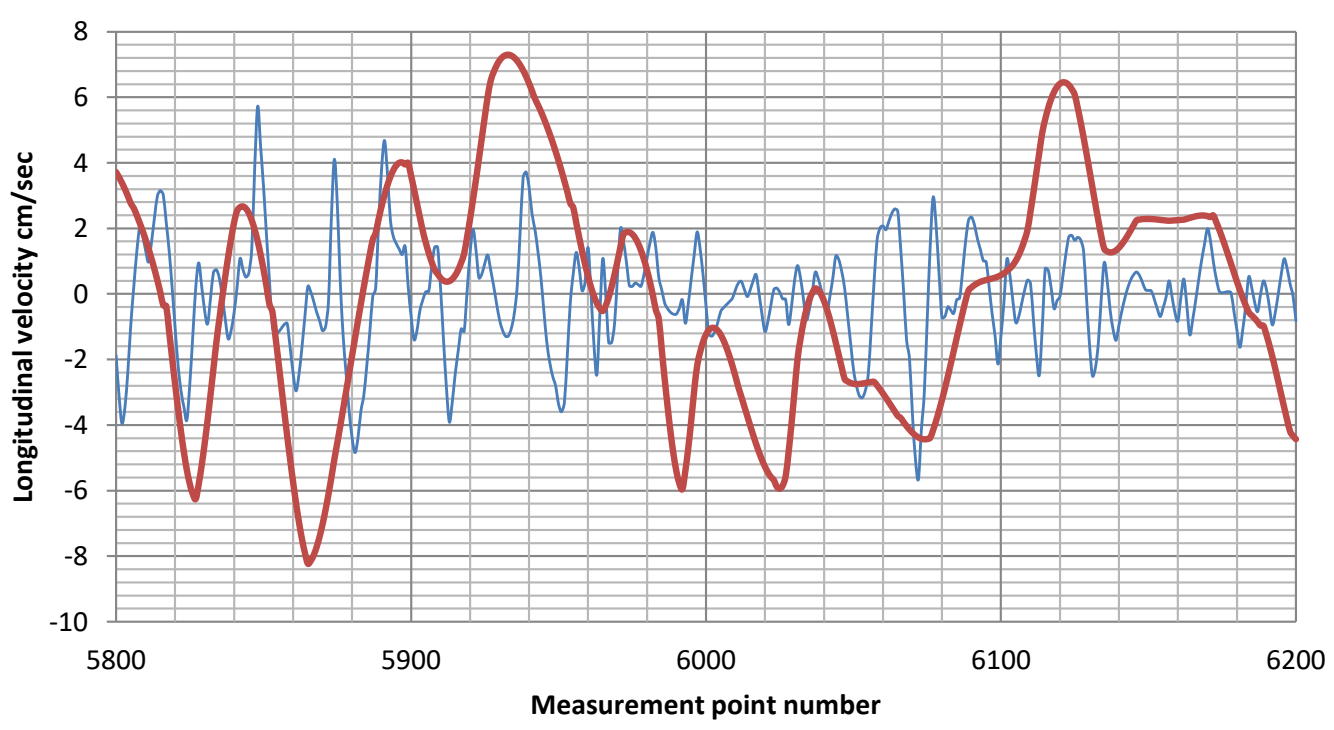

Fig. 8. Longitudinal velocity of separated groups of bottom vortices (thin line) and the summed velocities that are left after the separation of vortex structures (thick line) 


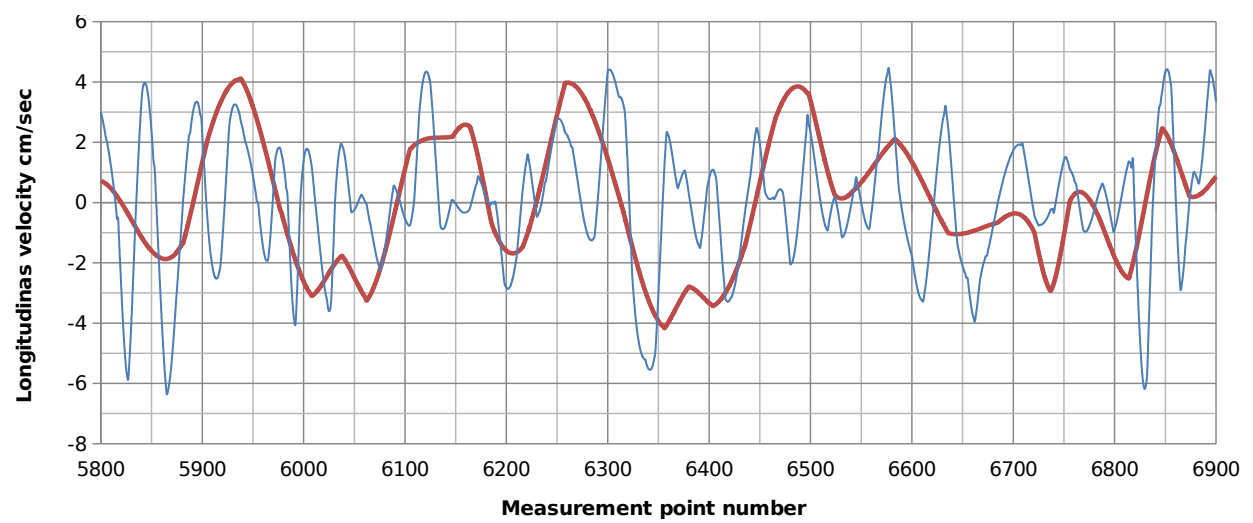

Fig. 9. Longitudinal velocity of separated single bank vortices, summed with accumulations of bottom vortex groups (thin line), and summed velocities of not separated bank vortex groups that were left and their accumulations (thick line)

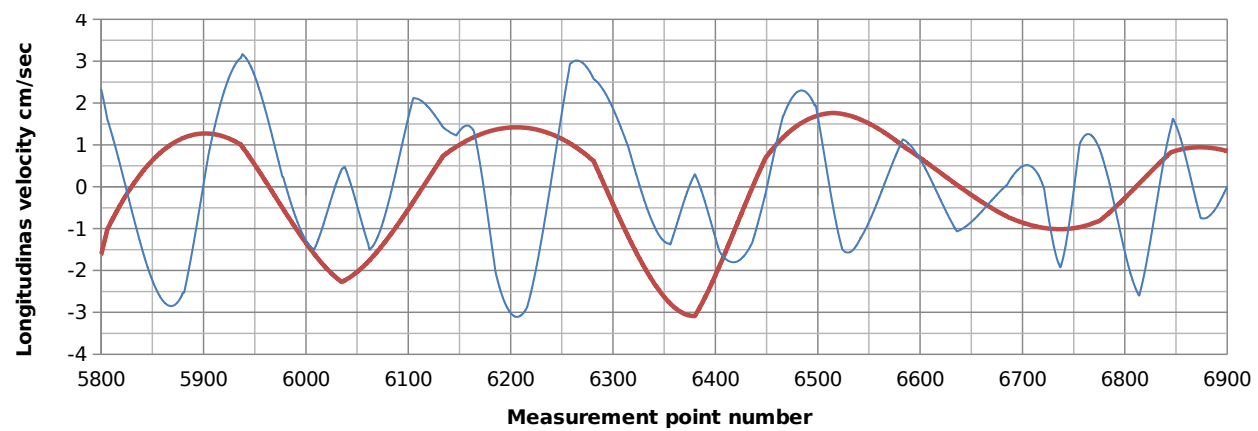

Fig. 10. Longitudinal velocity of separated groups of bank vortices (thin line) and the remaining accumulations of the groups

tices groups and their accumulations proceeded normally. Their separated velocities are shown in Fig. 10.

\section{Energy of Vortex Types}

The main characteristic of separated vortex structures is their kinetic energy level. This level was estimated as dependence on an energy relation of measured velocity and shear velocity. The average energy level of all the separated kinds of vortices was calculated along the all measurements (1-9000 points) according to the following formula:

$$
E=\frac{1}{8300} \sum_{j=350}^{8650} \frac{v_{j}^{2}}{u_{*}^{2}},
$$




$$
u_{*}=\sqrt{g R i}=2.29 \mathrm{~cm} / \mathrm{sec},
$$

where $E$ - the energy level, $v_{j}$ - velocity measured at point number $j, u_{*}$ - shear velocity.

The calculated energy intensities of all kinds of vortex structures, separated from the chronogram of measurements, are given in Table 1.

Table 1. Energy levels and frequencies of vortex structures

\begin{tabular}{|c|c|c|c|c|}
\hline & Kinds of vortex structures & $\begin{array}{c}\text { Energy } \\
\text { level in } \\
\text { floodplain }\end{array}$ & $\begin{array}{c}\text { Energy } \\
\text { level in } \\
\text { main channel }\end{array}$ & $\begin{array}{c}\text { Average } \\
\text { frequency in } \\
\text { floodplain } \\
1 / \mathrm{sec}\end{array}$ \\
\hline 1 & Horizontal macro whirlpools & 1.14 & & 0.25 \\
\hline 2 & Vertical macro whirlpools & 0.99 & 0.76 & 0.42 \\
\hline 3 & Vertical single vortices & 0.42 & 0.46 & 8.7 \\
\hline 4 & Groups of single vertical vortices & 0.66 & 0.29 & 2.4 \\
\hline 5 & Accumulations of vertical vortex groups & 0.73 & 0.39 & - \\
\hline 6 & Single bank vortices & 0.71 & - & - \\
\hline 7 & Groups of single bank vortices & 0.61 & - & 0.29 \\
\hline 8 & Accumulations of bank vortex groups & 0.67 & - & 0.25 \\
\hline 9 & Energy sum of all separated vortex kinds & 5.93 & 1.90 & \\
\hline 10 & $\begin{array}{l}\text { Energy estimated according to the chronogram } \\
\text { of measured velocities }\end{array}$ & 2.72 & 1.34 & \\
\hline
\end{tabular}

Table 1 shows investigation results for the energy levels of all vortices and their structures. In order to compare the energy of vortices in the main channel, the table shows and the main channel data. As can be seen, the horizontal and vertical macro whirlpools have the largest amounts of energy. This is because they are large main vortices, in which all the vortex types and their aggregates are generated and developed. However, the energy of macro whirlpools is much lower in the main channel as their development is decreased by the flow across the channel. Groups of vortices also have a larger amount of energy in floodplain. That is because the bottom vortices, generated in the floodplain at bottom unevenness, form regular rows and create a stronger stream around them. In the main channel, the vortices are not as regular because, at a flat bottom, they are generated more accidentally, thus becoming less regular.

The table presents the sum of energy level of all separated vortices and structures as well as the energy calculated from the chronogram of measured velocities. The latter is much lower. It shows the necessity of separation. In the main channel, this difference is not so large. Apparently, that is because the number of vortex kinds in the floodplain is greater. Therefore, the masking of vortex structures by unfavorable summation of their velocities is more intensive.

As already mentioned, single bank vortices could not be separated by the usual method. Therefore, their energy was estimated approximately. It was assumed that 
turning velocity relations at the bottom and at the bank are quite similar. According to the table data, the ratio of the energy of accumulations to the energy of their groups is $0.67 / 0.61=1.1$. It was assumed that the accumulations at the bottom are also 1.1 times greater than their groups, so the energy of the accumulations can be $1.1 \times 0.66=$ 0.73 . The common energy level of the single bank vortices and the accumulation of bottom vortex groups was 1.03. Investigations of the main channel revealed that the sum of the energy levels of all the separated vortex types was about 1.4 times greater than the value calculated as the sum of velocities in the measurement chronogram. The relation for these two vortex types in the floodplain may be similar. Then the sum of their energy would be $1.4 \times 1.03=1.44$ and the energy level of single bank vortices would be equal to $1.44-0.73=0.71$. These approximate values are given in Table 1.

\section{Frequency of Vortex Types}

During the separation of the graphs of vortices and their structures from the measurement chronogram, the average their frequency was also calculated. For that purpose, the number $n$ of the separated vortices or vortex types was calculated during the separation process. The interval of velocity measurement chronogram, employed for this process, was taken between the points 350 and 8650 , i.e. the length of the interval was 8300. Then, the frequency $f$ was estimated as follows:

$$
f=\frac{f_{m} n}{8300}
$$

where: $f_{m}=251 / \mathrm{sec}-$ frequency of measurement points.

As can be seen in Fig. 7 and Fig. 8, the frequency of single bottom vortices and their group's changes in somewhat not large diapason. The minimal frequency is about 4 times smaller than the average, and the maximal is about 4 times greater. The frequency of accumulations (Fig. 10) does not change and is equal to the frequency of macro whirlpools.

In the widely employed energy spectra method, frequencies of vortices are estimated by autocorrelation. It finds all the frequencies of vortices in the velocity measurement chronogram. However, accumulations of vortex groups make accidental velocity peaks in the chronogram and create a graph with non-existent frequencies, which are also detected by autocorrelation. Thus, the frequency graph becomes unrealistic. The separation of vortices does not allow it. This phenomenon is discussed in detail in the aforementioned article (Rimkus 2015).

\section{Review of Vortex Velocity Calculation Methods}

The above analysis of measurement data shows that the investigation of coherent structures of different kinds, obtained after their separation from the summary measurement graph, makes it possible to obtain more detailed and exact calculation and inves- 
tigation results and to avoid significant potential errors. The actually existing coherent structures of vortex groups and their accumulations have not yet been sufficiently investigated. Therefore, by the creation methods of investigations of these structures, it was mostly accepted that they all together form one large coherent structure. It cannot be investigated in detail, therefore, by the creation of the calculation methods, various assumptions, either exact or insufficiently exact, became necessary. An example of an unsuccessful method could be the previously mentioned energy spectra method. An example of a quite successful supposition is the turbulent stresses (Reynolds stresses). The formula for their calculation was deduced with the assumption that the velocities of turbulent flow are completely accidental (Agroskin et al 1950). Then it was accepted that the elementary liquid volume with the vertical velocity $v^{\prime}$ intertwines in the main stream, moving with the velocity $u^{\prime}$ ( $v^{\prime}$ and $u^{\prime}$ fluctuation velocities) and consequently, energy loses occur. Next formula for the calculation of the obtained turbulent stresses $\tau$ was developed:

$$
\tau=\rho u^{\prime} v^{\prime}
$$

where: $\rho$-density.

These turbulent stresses are included in Reynolds-averaged Navier-Stokes equations, which can be used to calculate, the average velocity distribution in the channel flow. However, the fluctuation velocities $u^{\prime}$ and $v^{\prime}$ are of course only the turning velocity components of turbulent vortices, which occur at the measurement point, i.e. the made assumption is unrealistic. However, the product of $u^{\prime}$ and $v^{\prime}$ is proportional to the kinetic energy of vortices. Therefore, it is also proportional and to the energy losses. That is because the Reynolds stresses could be approximately employed. This averaged Navier-Stokes equation was used for the calculation of the velocity field in a compound channel (Czernuszenko 2002). The calculated lines of constant velocity meanings were similar to those obtained by measurements, so the calculation method was accepted as suitable; however, they did not coincide closely, because the equation was not quite exact. It was made with suppositions. Nevertheless, it can give acceptable approximate results.

\section{Conclusions}

Turbulent vortices in the floodplain are more complicated than in the main channel, as they are generated not only at the bottom but also at the banks. Bottom vortices and their groups are thicker than those at the banks and make clear twists in the velocity measurement chronogram, what makes it possible to separate them. However, the largest bottom vortex structure, namely accumulations of vortex groups happened to have a similar thickness as single bank vortices, so formally they form a common structure. Consequently, they cannot be separated and It became necessary to use an approximate method, which produced less exact results. 
The main characteristic of turbulent vortices is their kinetic energy level. Vortices in the main channel and the floodplain have different amounts of energy. These vortices are lower in the channel, because the development of macro whirlpools in the channel is limited by square flow, which brings them out of the channel and they do not have enough time to fully develop.

The single bottom vortices and their groups are more regular in the floodplain, as they are generated at bottom unevenness with a stable place. On the flat bottom of the main channel, the points at which they are generated are not as stable, and the rows of vortex groups become not so strait. As a result, a weaker flow with a lower energy level develops around them.

To estimate the characteristics of vortices and their structures, i.e. of their oblique groups and accumulations, it was necessary to separate their velocities from the main measurement chronogram. The separated velocity graphs and their estimated characteristics are shown in Figs. 5-10 and in Table 1. In parallel to the separation process, the frequencies of the separated vortex types were also calculated. Their average values are also presented in Table 1.

It has been established by investigations that velocity measurement chronograms cannot always be used for necessary investigations because of an unsuccessful physical summation of velocities, created by all the vortex structures. The velocities get tangled by their summation. Therefore, the separation of vortex types is necessary and useful.

\section{Acknowledgment}

I am particularly indebted to prof. W. Czernuszenko for sharing his most recent data on the measurement of turbulence in a water laboratory in Warsaw, as well as for useful discussions.

\section{References}

Agroskin I. I., Dimitriev G. T., Pikalov F. I. (1950) Hydravlics, Gosenergoizdat.

Czernuszenko W., Kozioł A., Rowiński P. (2007) Measurements of 3D Structures in compound channel, Archives of Hydro-Engineering and Environmental Mechanics, 54 (1), 55-73.

Czernuszenko W., Rowinski P. (2008) Reynolds stresses in compound open channel flow-flume experiments, River flow 2008, Proceedings of the international conference on fluvial Hydraulics, Turkey, September 3-5, 2008, 289-297.

Grishanin K. V. (1969) Dynamics of river flow, Gidrometeorologicheskoeizdatelstvo, Leningrad, pp. 428 (In Russian).

Klaven A. B. (1966) Investigation on the structure of turbulent flow, Trudy Gosudarstvennovo Gidrologicheskovo Instituta, Vypusk 136, 65-76 (In Russian).

Nezu I., Azuma R. (2004) Turbulence characteristics between particles and fluid in particle-laden open channel flows, Journal of Hydraulic Engineering, 130 (10), 988-1001.

Nezu I., Nakahawa H. (1993) Turbulence in open channels, IAHR Monograph series, A.A. Balkema, Rotterdam, The Netherlands. 
Rimkus A. (2012) Structure of turbulent vortices in a compound channel, Archives of Hydro-Engineering and Environmental Mechanics, 59 (3-4), 113-135.

Rimkus A. (2015) Structures of turbulent vortices and their influence on flow properties, Journal of water Security, 1 (1), 14-24.

Vanoni V. A. A., Nomicos G. N. (1959) Resistant properties of sediment-laden streams, J. Hydr. Div. Am. Soc. Civ. Engrs., 85, No. HY5. 